\title{
Simulación del proceso de concentración de aceite esencial de Cidrón (Lippia citriodora) por destilación molecular de película descendente
}

CHEMICAL ENGINEERING

\section{Simulation of the concentration process of Lippia citriodora (Cidrón) essential oil using falling - film molecular distillation model}

\author{
Ivonne X. Cerón*, Carlos A. Cardona**§, Luis A. Toro*** \\ * Departamento de Ingeniería Química, Instituto de Biotecnología y Agroindustria, Universidad \\ Nacional de Colombia sede Manizales, Manizales, Colombia. \\ ** Departamento de Matemáticas y Estadística, Instituto de Biotecnología y Agroindustria, \\ Universidad Nacional de Colombia sede Manizales, Manizales - Colombia.ccardonaal@unal.edu.co§ \\ ***Departamento de Física y Matemáticas, Universidad Autónoma de Manizales \\ Manizales - Colombia. \\ ixcerons@unal.edu.co,ccardonaal@unal.edu.co,latoroc@unal.edu.co,
}

(Recibido: Marzo 11 de 2011 -Aceptado: Mayo 7 de 2012 -Versión Final: Junio 19 de 2012)

\begin{abstract}
Resumen
En los procesos para la producción y purificación de productos térmicamente inestables, el diseño juega un papel importante ya que permite determinar los costos y la calidad del producto obtenido. En este trabajo se presenta el modelo de un destilador molecular de película descendente, el cual tiene en cuenta las ecuaciones de balance de calor, materia y cantidad de movimiento para una mezcla multicomponente, aplicado a la concentración del aceite esencial de cidrón (Lippia citrodora). El sistema de ecuaciones diferenciales acopladas resultantes se resolvió mediante el método de Crank-Nicholson. El modelo así obtenido permite simular el proceso de destilación molecular y se utilizó para obtener los perfiles temperatura y concentración en las películas de evaporación y condensación. El aceite esencial se logró concentrar de $47.79 \%$ a $89.21 \%$ de citral por fondos y de $41.62 \%$ a $72.23 \%$ de limoneno por destilado.
\end{abstract}

Palabras Claves: Destilador molecular, Aceite esencial de cidrón, Simulación, Mezclas multicomponentes.

\begin{abstract}
In the processes of production and purification of thermally unstable products, the design plays an important role since it determines the costs and quality of the obtained product. In this work, we present a mathematical model for a falling-film molecular distiller based on the equations of balance of heat, material and momentum for a multicomponent mixture, which is applied to the concentration of Lippia citrodora essential oil. The model consisted of coupled differential equations that were solved using the Crank-Nicholson method for obtaining the profiles of temperature and molar fractions of the components in the evaporation and condensation surfaces. The essential oil is obtained with concentrations from $47.79 \%$ to $89.21 \%$ of citral in the bottoms and $41.62 \%$ to $72.23 \%$ of limonene in the top.
\end{abstract}

Keywords: Molecular distillation, Lippia citrodora essential oil, Simulation, Multicomponent mixtures. 


\section{Introducción}

Para el desarrollo de un proceso industrial se requiere un estudio experimental de las condiciones que afectan la rentabilidad del proceso y la calidad del producto. Sin embargo, estas pruebas implican altas inversiones de dinero y tiempo, lo cual puede impedir el desarrollo de una investigación. Esto puede ser rápidamente solucionado mediante el uso de métodos matemáticos, que permiten estudiar la influencia de diferentes variables en el proceso. De esta manera, se asegura una reducción de los costos y de los consumos de masa y energía, así como la fiabilidad de los resultados en un tiempo mínimo. Esto es particularmente relevante en el caso del proceso de destilación molecular, que tiene altos costos energéticos, principalmente por ser una operación de alto vacío, inferior a una presión absoluta de 10-5bar según Martinello et al. (2003).

En el proceso de destilación molecular la mezcla a tratar se pone en contacto con una superficie caliente (superficie de evaporación) de manera que se forma una película delgada, donde las moléculas incrementan su energía interna y el compuesto más volátil comienza a evaporarse y choca contra una superficie fría (superficie de condensación). La eficiencia de este proceso se basa en la reducida distancia entre la superficie de condensación y la superficie de evaporación, la cual debe ser menor que la trayectoria libre media de las moléculas de la mezcla a tratar y también en el alto vacío que caracteriza a la operación. Según Cardona et al. (2005), la trayectoria libre media es la distancia que la molécula puede viajar antes de chocar con otra molécula o con la pared de un contenedor. Como las moléculas en fase vapor deben recorrer una distancia menor que su trayectoria libre media, no colisionan entre sí en su trayecto desde la superficie de evaporación hasta la de condensación.

En la superficie de evaporación ocurre una transferencia simultánea de masa y calor, existiendo una variación de concentración y temperatura en la película. Martins et al. (2006); Cermak et al. (2007); Martinello et al. (2007): Shao et al. (2007) han llevado a cabo estudios teóricos y experimentales para determinar el rendimiento de los procesos de destilación molecular para distintas aplicaciones. Entre los avances en el modelado teórico de esta operación, se encuentra el aporte de Bose \& Palmer (1984), quienes mostraron la existencia de gradientes de concentración y temperatura en la fase líquida y su influencia en la eficiencia de separación. Bhandarkar \& Ferron (1991) trabajaron en la transferencia de masa y calor en una película líquida sobre un evaporador cónico centrífugo. Batisella \& Maciel (1996), reportan resultados comparativos de la eficiencia y rendimientos entre destilación molecular de película descendente y centrífugos. Chang et al. (1997) y Micov et al. (1997) analizaron la eficiencia del destilador molecular mediante un modelo desarrollado para mezclas binaras.

La presión de trabajo del proceso de destilación molecular, permite operar a bajas temperaturas y obtener altas volatilidades relativas de los componentes, convirtiéndose en una operación factible para separar compuestos que se degradan fácilmente con la temperatura. Por tal razón, este proceso es ideal para la purificación de productos biológicos con aplicaciones en la industria cosmética, farmacéutica y de alimentos entre otras. Tal es el caso del aceite esencial de cidrón (Lippia citriodora), el cual fue estudiado por Argyropoulou et al. (2007) reconociendole propiedades antiespasmódicas, digestivas, expectorantes, sedativas, antihistamínicas, analgésicas y antipiréticas. Investigaciones realizadas por Carnat et al. (1999); Argyropoulou et al. (2007); Ragone et al. (2007); Bilia et al. (2008) han encontrado grandes diferencias en la composición química del aceite esencial de las hojas de L. citriodora, identificando compuestos fenólicos (flavonoides y ácidos fenólicos) . Argyropoulou et al. (2007), identificó en los aceites esenciales de las flores y hojas de L. citriodora, cultivadas en Grecia, geranial (38.7\%), neral $(24.5 \%)$ y limoneno $(5.8 \%)$, como principales componentes. Los aceites esenciales de L. citriodora, cultivados en Chile, según Carnat et al. (1999), son mayoritarios en citral (geranial + neral), representado el $41 \%$ del total del aceite, otros constituyentes principales fueron el limoneno, el cineol, y el citronelol, todos presentes con niveles mayores al 5\%. Cerón (2009), encontró que el componente principal del aceite esencial de cidrón cultivado en Colombia es 
el citral con un $46.5 \%$, limoneno y $\alpha$-pineno con un $40.5 \%$ y $10.3 \%$, respectivamente. Según Vekiari et al. (2001), esto se debe a que los aceites esenciales varían su composición y calidad de acuerdo a diferentes factores como el clima, composición del suelo, órgano de la planta, edad y etapa del ciclo vegetativo. Para la industria de aromas, aparecen múltiples problemas en la producción de extractos, principalmente porque los aceites esenciales están compuestos de hidrocarburos terpénicos y en menor proporción de compuestos oxigenados. Estos terpenos son parcialmente saturados y químicamente inestables, propensos a las agrupaciones estructurales en presencia de aire, luz y agua. Del mismo modo, estas reacciones se aceleran por el calor y bajos $\mathrm{pHs}$.

El propósito de este trabajo es evaluar el modelo matemático de un destilador molecular de película descendente para una mezcla multicomponente aplicado al aceite esencial de cidrón, con el fin de proporcionar una herramienta matemática para la simulación del proceso.

\section{Aproximación Metodológica}

Para el desarrollo del modelo matemático se consideraron las composiciones normalizadas de los principales compuestos del aceite esencial de cidrón: limoneno, citral y $\alpha$-pineno con una concentración $41.62 \%, 47.79 \%$ y $10.59 \%$ respectivamente reportados en Cerón (2009). El propósito es remover el limoneno, que al ser más volátil, se recupera en el destilado del aceite. Este terpeno es muy inestable y se oxida con facilidad, afectando la calidad del aceite.

\subsection{Equilibrio de fases a baja presión}

El equilibrio líquido vapor a presiones subatmosféricas, es utilizado para analizar las variables del proceso de destilación molecular. El tratamiento matemático del ELV comprende el cálculo de equilibrio de fases a baja presión, teniendo en cuenta que la fase vapor y la fase líquida se encuentran en equilibrio a la misma temperatura y presión, cumpliéndose que la fugacidad del líquido del componente i es igual a la fugacidad del vapor del componente i, por lo tanto:

$$
f_{i}^{(V)}=f_{i}^{(L)}
$$

La predicción del ELV a baja presión de los compuestos principales del aceite esencial de cidrón (citral, limoneno, $\alpha$-pineno), se realizó teniendo en cuenta que a bajas presiones las interacciones entre moléculas de distintas especies en la fase vapor disminuyen, permitiendo suponer que la fase vapor tiene un comportamiento de gas ideal y en la fase líquida se tienen en cuenta las desviaciones con respecto a la solución idealizada. Para el cálculo del ELV, es necesario conocer los parámetros termodinámicos de los compuestos, los cuales fueron estimados utilizando el método descrito por Marrero \& Gani (2001), donde se proponen tres niveles para realizar la estimación de las propiedades de los componentes puros. E1 ELV se modeló utilizando el lenguaje de programación MatLab R2001b.

\subsection{Modelo de Destilación molecular}

En la Figura 1 se muestra en forma esquemática el funcionamiento de un destilador molecular de película descendente. Las ecuaciones de transporte que describen el proceso que tiene lugar en la película de evaporación surgen del planteo de los balances de cantidad de movimiento, de masa y de energía que han sido reportados por Micov et al. (1997); Lutisan et al. (2002); Sales-Cruz \& Gani (2006). La operación de destilación molecular se lleva a cabo en estado estacionario. Asumiendo que no existe un proceso de re-evaporación de las moléculas desde la superficie y que las colisiones entre las moléculas son despreciables, se justifica la baja presión de trabajo y se asegura que la trayectoria libre media de las moléculas sea mayor que la distancia entre las dos superficies como ha sido expuesto por Lutisan \& Cvengros (1995).

Los fenómenos de transferencia de cantidad de movimiento es explicado por la ecuación de Navier-Stokes para flujo en la película por gravedad y los fenómenos de calor y masa por la ecuación de balance térmico y de difusión, respectivamente y descritos por Lutisan et al. (2002). Para flujo pelicular en la película de 


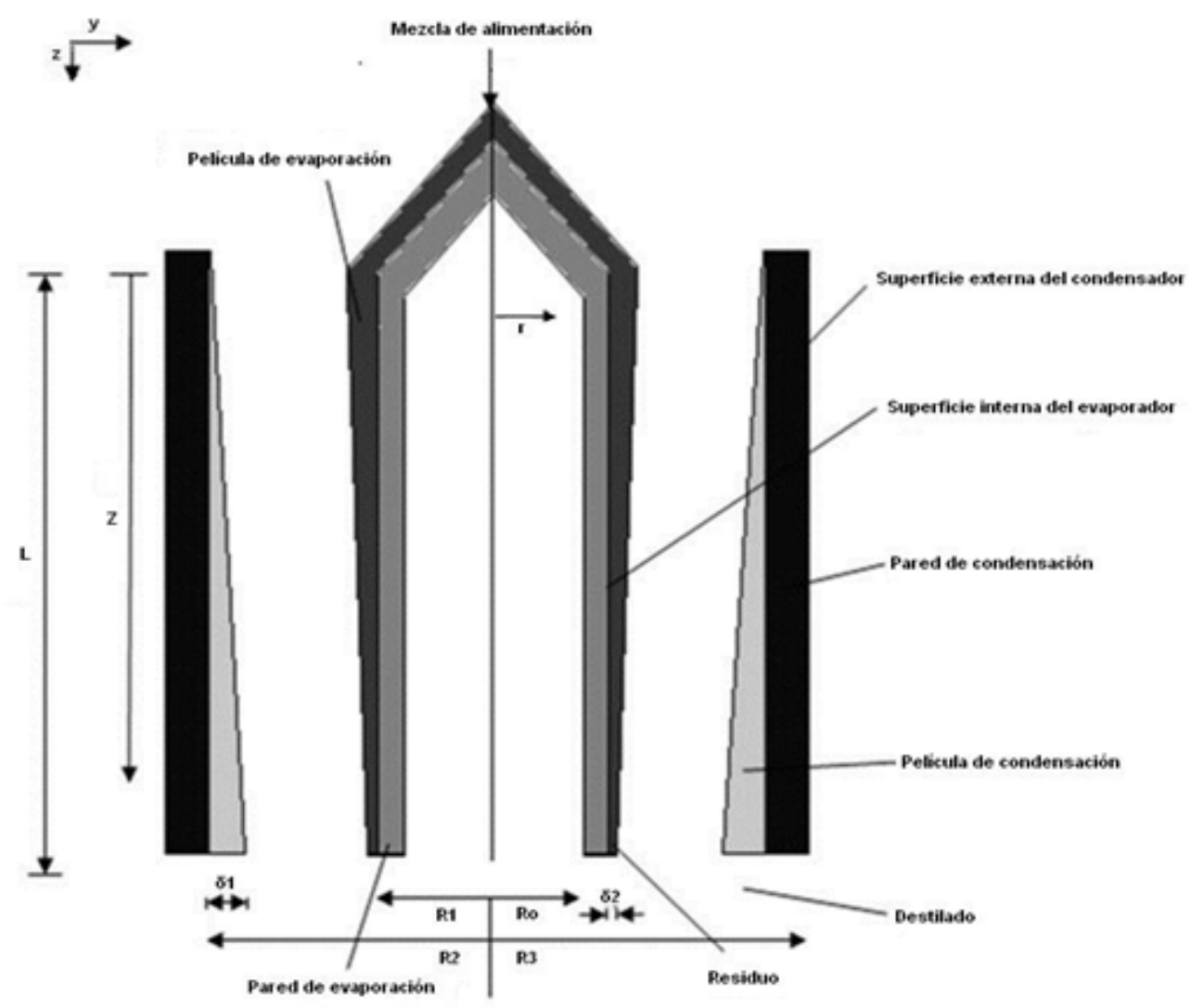

Figura 1. Esquema de un equipo de destilación molecular

gravedad se expone la ecuación de Navier Stokes de la siguiente manera:

$$
\frac{D v_{j}}{D t}=g+\vartheta \nabla^{2} v_{j}
$$

Cvengros et al. (2000) postula que la velocidad tiene solamente componente axial y depende sólo de la posición radial, con lo cual la ecuación (2) se convierte en una ecuación diferencial de segundo orden:

$$
\frac{\vartheta}{r} \frac{d}{d r}\left(r \frac{d v_{z, j}}{d r}\right)+g=0
$$

Expresando en coordenadas cartesianas, y teniendo en cuenta la solución de Nusselt para el perfil de velocidades se obtiene:

$$
v_{z}(y, z)=\frac{g h_{1}^{2}}{v}\left(\frac{y}{h_{1}}-\frac{y^{2}}{h_{1}^{2}}\right)
$$

La ecuación de balance de energía es:

$$
v_{z} \frac{d T(y, z)}{d z}=\beta\left(\frac{d^{2} T}{d y^{2}}+\frac{d^{2} T}{d z^{2}}\right)
$$

Donde:

$$
\beta=\frac{1}{C p_{l i q} \rho}
$$

Lutisan et al. (2002) explica que el calor se transfiere principalmente por convección a lo largo de la dirección z, por lo tanto el término $d^{2} T$ / $d z^{2}$ es muy pequeño en comparación con los otros dos términos Lutisan et al. (2002). Entonces la Ec. (5) puede ser escrita de la siguiente manera:

$$
v_{z} \frac{d T(y, z)}{d z}=\beta\left(\frac{d^{2} T}{d y^{2}}\right)
$$


Con las condiciones iníciales Ec. (8), condición límite en la pared del evaporador Ec. (9) y de frontera en la interfase de la película Ec. (10):

$$
\begin{gathered}
T(y, 0)=T_{F} \\
T(0, z)=T_{w} \\
\Gamma \frac{d T}{d y}=-\Delta H_{e v p} k \text { en } y=h_{1}
\end{gathered}
$$

Donde:

$$
k_{i}=\frac{x_{i} p_{i}^{0}\left(T_{s}(z)\right)}{\sqrt{2 \pi M_{i} T_{i}\left(y, h_{1}\right)}} i=\text { componente }
$$

$k_{i}$ es la velocidad en la superficie de evaporación para un líquido ideal expuesto por Nguyen \& LeGoffic (1997). La dependencia del flujo $I$ en coordenada $\mathrm{z}$ es dada por la ecuación de continuidad:

$$
\frac{d I_{i}(z)}{d z}=-2 \pi r k_{i}
$$

Con la condición inicial: $I_{i}=F_{i}$

El conjunto de Ec. (7) y Ec. (12) con las condiciones límite especificadas por las Ec. (9) y Ec. (10) se resuelve numéricamente. Para $0=z$ se determina las condiciones límite Ec. (8) y Ec. (13). En cada paso $\Delta z$, el valor del flujo se establece a partir del valor de $I$ calculado en previos pasos, utilizando la aproximación de Taylor de la Ec. (12) y siguiendo el proceso descrito por Cvengros et al.(2000).

$$
I(z+\Delta z)=I(z)-2 \pi r k \Delta z
$$

El espesor de la película se calcula a partir del valor conocido de $I(z+\Delta z)$

$$
h_{1}(z+\Delta z)=\sqrt[3]{\frac{3 v}{2 \pi r g c} I(z+\Delta z)}
$$

\section{Ecuación de balance de masa}

La transferencia de masa se rige por la ecuación de difusión explicada por Lutisan et al. (2002):

$$
\frac{D C_{i j}}{D t}=D_{i j} \nabla^{2} C_{i j}
$$

Expresando la ecuación anterior en función de las concentraciones y simplificando para condiciones de estado estable se obtiene:

$$
v_{z} \frac{d C_{i}(y, z)}{d z}=D_{i}\left(\frac{d^{2} C_{i}}{d y^{2}}\right)
$$

La Ec. (16), se resuelve con las condiciones iníciales y condiciones limite:

$$
\begin{gathered}
C_{i}(y, 0)=C_{i F} \\
\frac{d C_{i}(0, z)}{d y}=0 \\
D_{i} \frac{d C_{i}\left(h_{1}, z\right)}{d y}=I_{i}
\end{gathered}
$$

El determinación del perfil de la película de condensado, se realiza a partir de las condiciones de frontera espacio de destilación - película de condensado, como la temperatura de superficie, flujo y fracción molar de cada uno de los compuestos que se evaporan. Las ecuaciones de balance de masa y energía son aplicadas para la película de condensado.

\subsection{Simulación del modelo de destilación molecular}

Las derivadas parciales de primero y segundo orden que componen las ecuaciones de balance de materia y energía pueden aproximarse mediante ecuaciones de diferencias finitas. Se utilizó el esquema implícito de Crank-Nicholson, basado en el método de diferencias finitas, expresando la derivada de un punto intermedio E (Figura 2) de la retícula y expuesto por Cardona et al. (2005). Aplicando este concepto se obtiene las aproximaciones para la variación cartesiana de la temperatura y concentración en cada nodo usando las condiciones iníciales y de frontera. 
La ecuaciones diferenciales parciales Ec. (7) y Ec. (17), con las condiciones límite Ec. (9), Ec. (10) y Ec. (19), Ec. (20) respectivamente, se pueden resolver fácilmente si se representan en ecuaciones diferenciales ordinarias usando la aproximación del método de mallas. El valor de la temperatura en el nodo $(m, n)$, (Figura 2), se calcula a partir del conjunto de ecuaciones lineales: $\operatorname{Ec}(21), \operatorname{Ec}(22)$

Las condiciones iníciales son dadas al conocer el perfil de temperaturas en la primera fila (para $0=n$ ), de acuerdo a la Ec. (8). Las condiciones límite en la pared del evaporador se especifican por la Ec. (9). Estas condiciones diferenciales límites se remplazan por una diferencia expresando la Ec. (23), obteniendo un conjunto de ecuaciones diferenciales ordinarias y varias ecuaciones no lineales que pueden ser resueltas utilizando el método de Runge-Kutta.

El valor de la concentración en el nodo $(m, n)$ (Figura 2), se calcula a partir del conjunto de ecuaciones lineales: Ec (24), Ec (25).

$$
\begin{gathered}
\frac{-A}{2} T_{m-1 . n}+(1-A) T_{m, n}-\frac{A}{2} T_{m+1 . n}=\frac{A}{2} T_{m+1 . n-1}+(1-A) T_{m, n-1}+\frac{A}{2} T_{m-1 . n-1} \\
A=\frac{\vartheta \Delta z}{v_{z} \Delta y^{2}} \\
T_{s}(z)=T_{w}-h_{1} \frac{\Delta H_{e v p} k\left(T_{s}(z)\right)}{\Gamma} \\
\frac{-B}{2} C_{i m-1 . n}+(1-B) T_{m, n}-\frac{B}{2} C_{i m+1 . n}=\frac{B}{2} C_{i m+1 . n-1}+(1-B) C_{i m, n-1}+\frac{B}{2} C_{i m-1 . n-1} \\
B=\frac{D_{i} \Delta z}{v_{z} \Delta y^{2}}
\end{gathered}
$$

Las condiciones iníciales son dadas al conocer el perfil de concentraciones en la primera fila (para $0=n)$, de acuerdo a la Ec. (18). Las condiciones limite en la pared del evaporador se especifican por la Ec. (19) y en la interfase pared-espacio de destilación por la Ec. (20), las cuales se remplazan por una diferencia, expresando la Ec. (26) y Ec. (27), respectivamente.

$$
\begin{gathered}
C_{i_{m+1, n}}=C_{i_{m-1, n}} \\
C_{i_{m_{\max }, n}}=C_{i_{m_{\max -1, n}}}-\frac{h_{1} I_{i}}{D_{i}}
\end{gathered}
$$

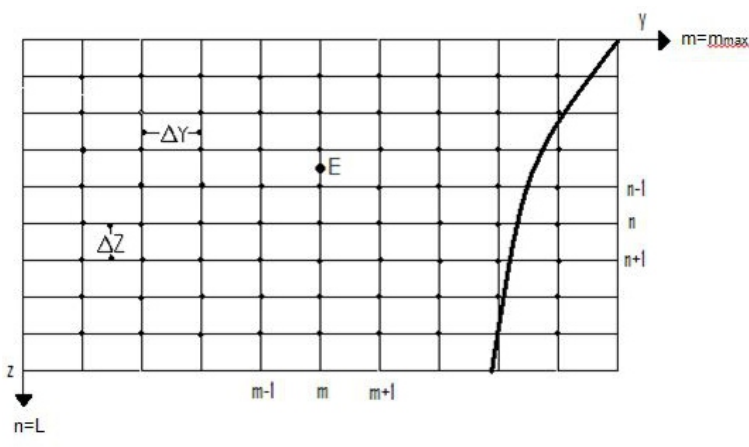

Figura 2. Retícula para el análisis de película

Para resolver las ecuaciones es necesario seguir una secuencia lógica que integra el orden y el método de solución de todas las ecuaciones 
involucradas. Las ecuaciones se resolvieron en el lenguaje de programación MatLab R2010b, siguiendo el algoritmo mostrado en la Figura 3.

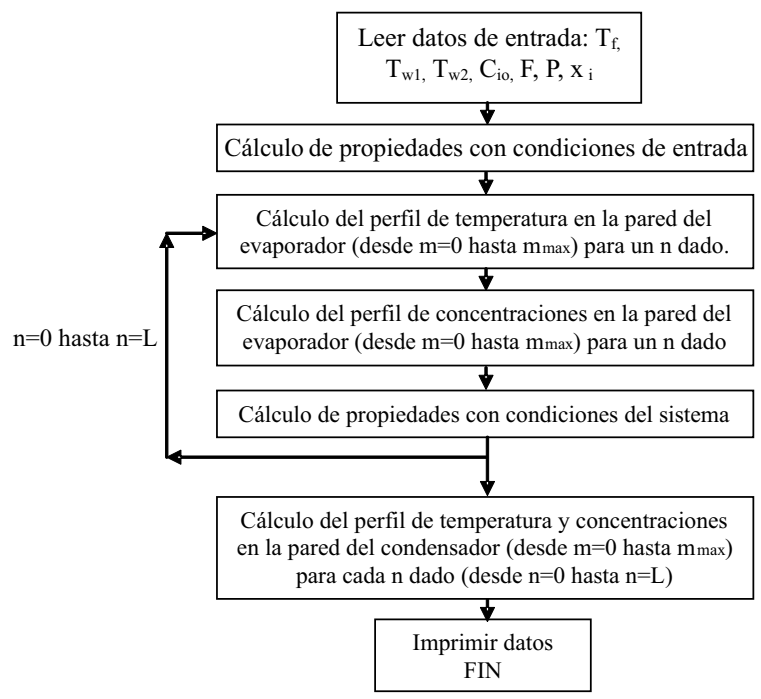

Figura 3. Algoritmo para la solución del modelo matemático de un destilador molecular de película descendente

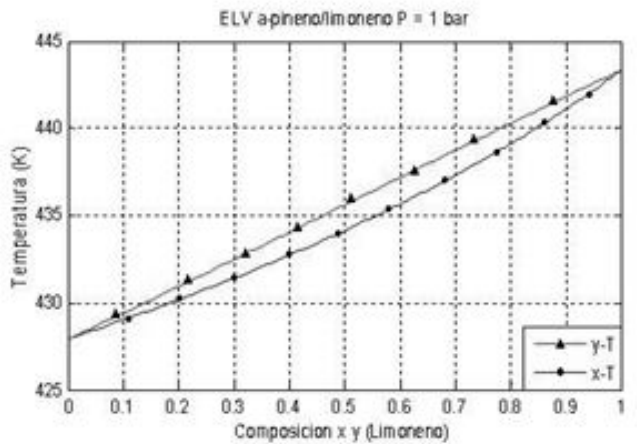

\section{Resultados y Discusión}

\subsection{Equilibrio de fases}

En la Figura 4 se presenta el equilibrio binario a 1 bar y en la Figura 5 se muestra el equilibrio líquido vapor a $0.001 \mathrm{mbar}$ para los tres componentes considerados agrupados de a pares, que es la presión de trabajo en el proceso de destilación molecular. Al disminuir la presión, la volatilidad relativa mejora permitiendo una mejor separación de los compuestos analizados.

El equilibrio binario es útil para definir las condiciones de operación del proceso de destilación molecular, como la temperatura de evaporación y la temperatura de condensación.

\subsection{Perfiles del proceso de destilación molecular}

La mezcla ternaria de los compuestos principales de aceite esencial de cidrón fue concentrada siguiendo la metodología mostrada en la Figura 3, obteniéndose un rendimiento de la operación para

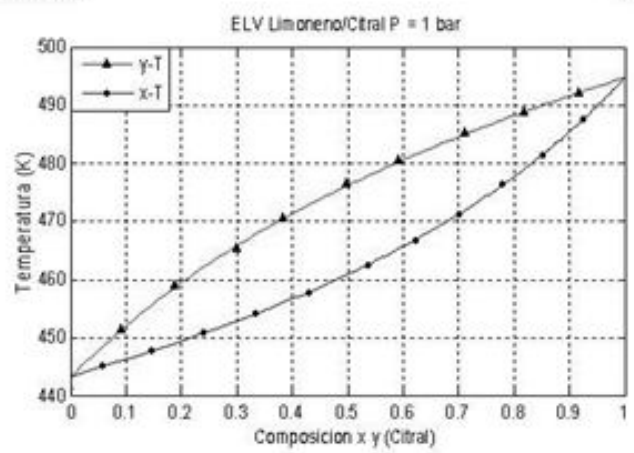

Figura 4. Equilibrio líquido vapor a 1 bar de los principales compuestos del aceite esencial de cidrón 

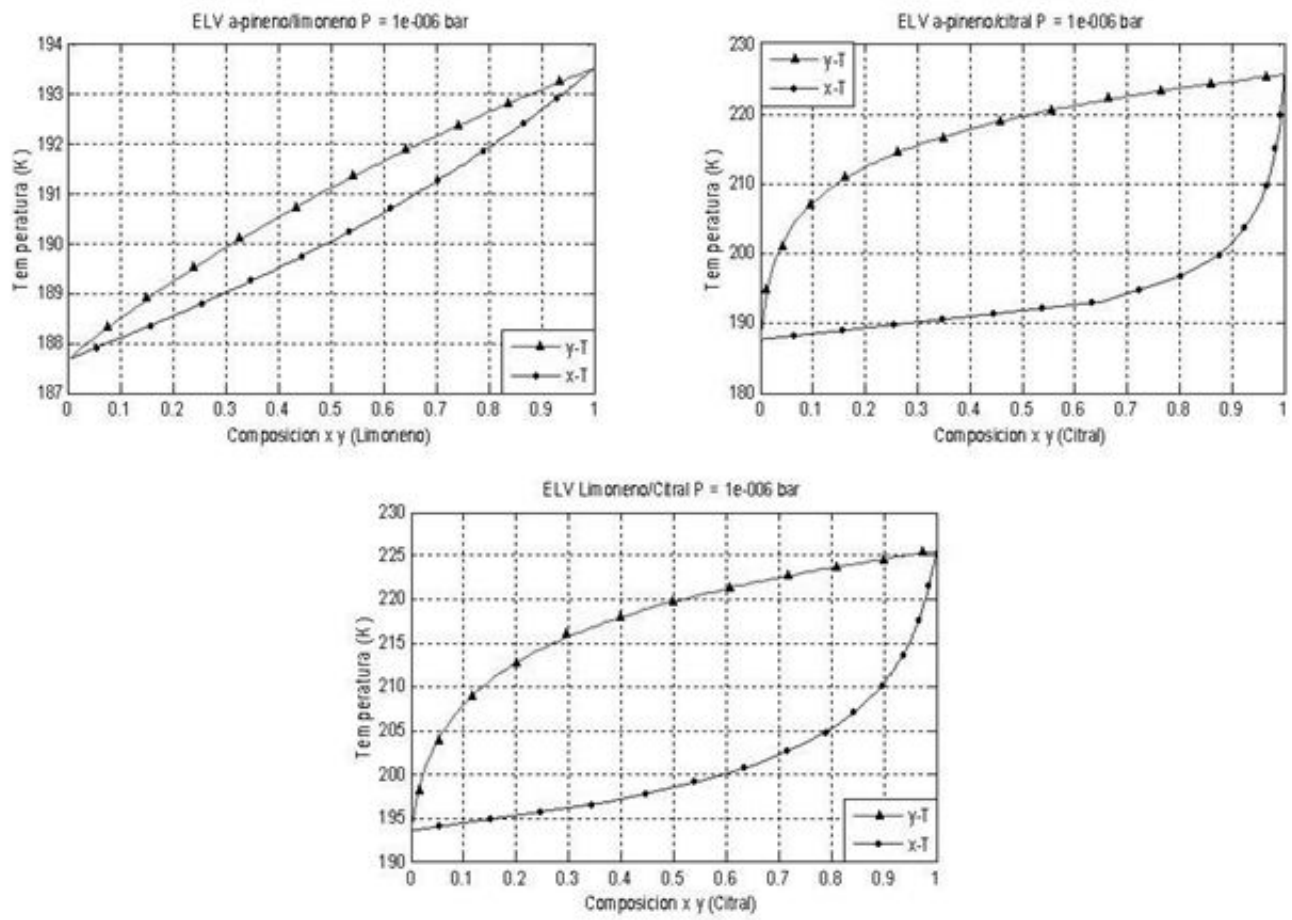

Figura 5. Equilibrio líquido vapor a 0.001 mbar de los principales compuestos del aceite esencial de cidrón

del $92 \%$ para concentrar el citral en el aceite esencial de cidrón. Se simuló el proceso de destilación molecular, con los parámetros mostrados en la Tabla 1 .

El citral fue concentrado en la corriente de fondos a $89.21 \%$ (fracción molar) mientras que en el destilado se obtuvo una concentración de $6.82 \%$, a una presión de 54 pascales y una temperatura de fluido de refrigeración y calentamiento de $230 \mathrm{~K}$ y $250 \mathrm{~K}$, respectivamente. Esto quiere decir que el aceite esencial rico en citral obtenido por fondos, es más estable que el aceite de cidrón alimentado dado a su baja concentración de limoneno $(10.36 \%)$ y que el aceite esencial fue concentrado 13 veces la concentración de alimentación de citral.

La Figura 6 muestra la variación de la temperatura en la película de evaporación a lo largo del destilador molecular. La temperatura en la superficie de la película, representa un factor determinante para el proceso de evaporación ya que de esta depende la velocidad de evaporación de las mezclas bajo estudio. La temperatura decae en los primeros centímetros de la longitud del evaporador, debido a que la mezcla necesita energía interna para poder iniciar la evaporación.

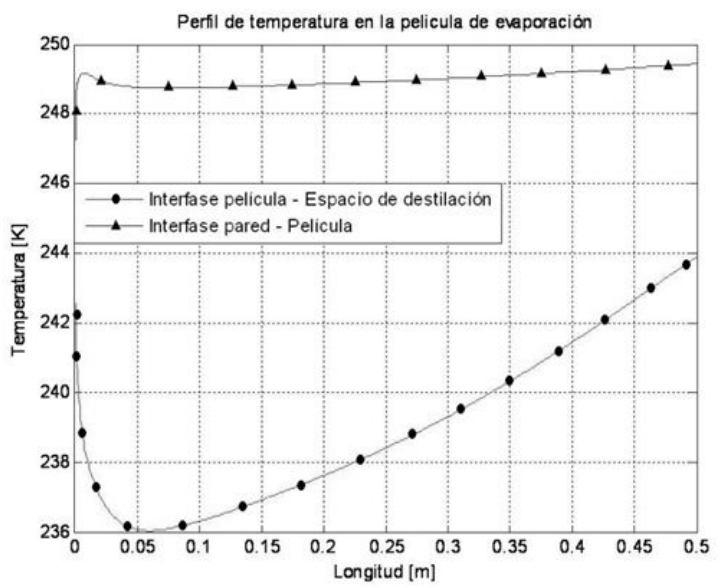

Figura 6. Perfil de temperaturas en la película de evaporación

En la Figura 7 se presenta la variación del espesor de la película de evaporación a lo largo de la longitud del condensador. El espesor de la película 
Tabla 1. Variables de entrada y datos generados en la solución del modelo

\begin{tabular}{|c|c|}
\hline \multicolumn{2}{|c|}{ Información de la operación "Aceite esencial de cidrón" } \\
\hline Número de componentes de la mezcla & 3 \\
\hline Fracción molar de $\alpha$ - pineno & 10.59 \\
\hline Fracción molar de citral & 47.79 \\
\hline Fracción molar de limoneno & 41.62 \\
\hline Radio del evaporador $[\mathrm{m}]$ & 0.05 \\
\hline Radio del condensador [m] & 0.0627 \\
\hline Espacio entre las paredes del equipo [m] & 0.0127 \\
\hline Longitud de la pared $[\mathrm{m}]$ & 0.5 \\
\hline Temperatura de fluido de refrigeración $[\mathrm{K}]$ & 230 \\
\hline Temperatura de fluido de calentamiento $[\mathrm{K}]$ & 250 \\
\hline Temperatura de alimentación $[\mathrm{K}]$ & 245 \\
\hline Número de incrementos en " $y$ " en las películas & 10 \\
\hline Número de incrementos longitudinales & 1,000 \\
\hline Delta Z [m] & $7 e-4$ \\
\hline Espesor inicial de la película de evaporación & $2.97 \mathrm{e}-4$ \\
\hline Caudal de alimentación $[\mathrm{ml} / \mathrm{s}]$ & 4.3633 \\
\hline Presión de operación [pa] & 54 \\
\hline \multicolumn{2}{|l|}{ Resultados } \\
\hline Flujo de fondos $[\mathrm{ml} / \mathrm{s}]$ & 2.143 \\
\hline Fracción molar de $\alpha$-pineno & 0.43 \\
\hline Fracción molar de citral & 89.21 \\
\hline Fracción molar de limoneno & 10.36 \\
\hline Flujo de destilado $[\mathrm{ml} / \mathrm{s}]$ & 2.215 \\
\hline Fracción molar de $\alpha$-pineno & 20.95 \\
\hline Fracción molar de citral & 6.82 \\
\hline Fracción molar de limoneno & 72.23 \\
\hline
\end{tabular}

decrece fuertemente con el incremento de la temperatura debido a la temperatura de alimentación y la viscosidad de la mezcla que son factores de influencia en la evaporación.
El perfil de composiciones de la película de evaporación se presenta en la Figura 8 . Se observa un descenso en la concentración de los componentes más volátiles: $\alpha$-pineno y limoneno 


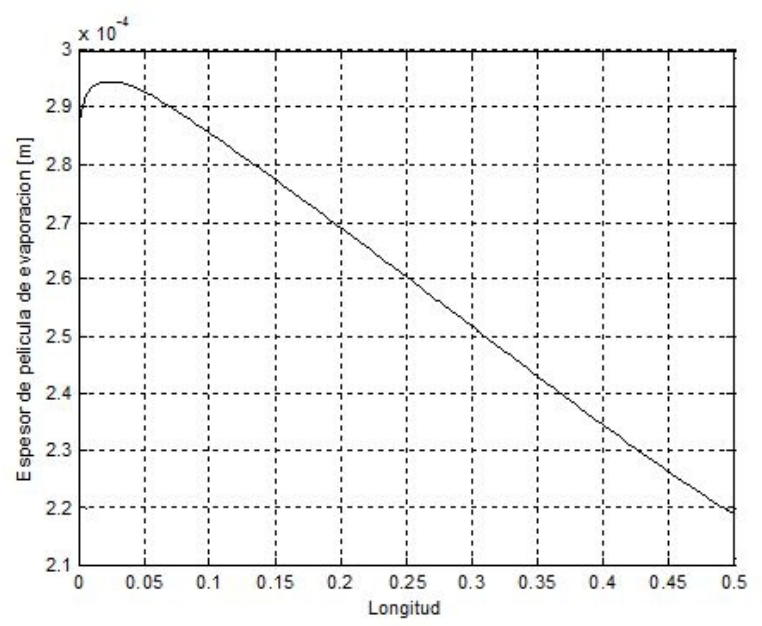

Figura 7. Espesor de la película de evaporación

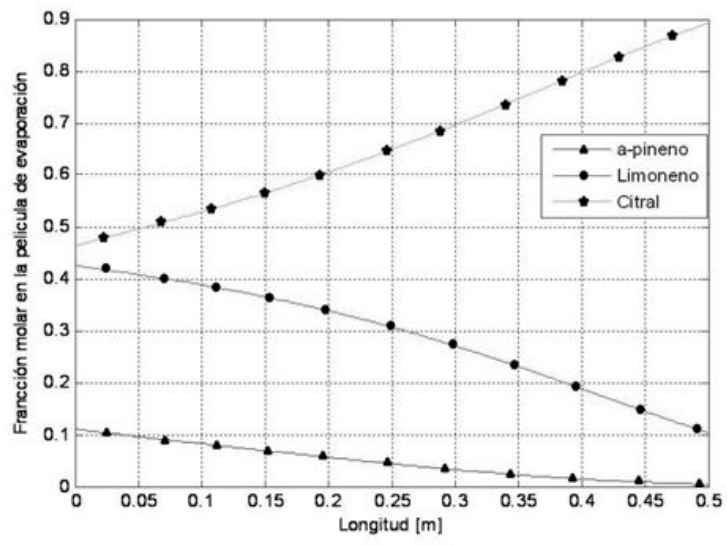

Figura 8. Perfil de composiciones de la película de evaporación

y un aumento en la composición del citral, que es el principal componente responsable del olor y sabor del aceite esencial de cidrón, cumpliéndose el objetivo de concentrar el aceite esencial.

A lo largo del evaporador la mezcla va perdiendo masa que se evapora y pasa a la película de condensación. En la Figura 9 se muestra el comportamiento del flujo el cual va decreciendo rápidamente.

La Figura 10, presenta el perfil de temperaturas en la película de condensación, la temperatura en la superficie aumenta debido a la ganancia de energía por la mezcla que se evapora.

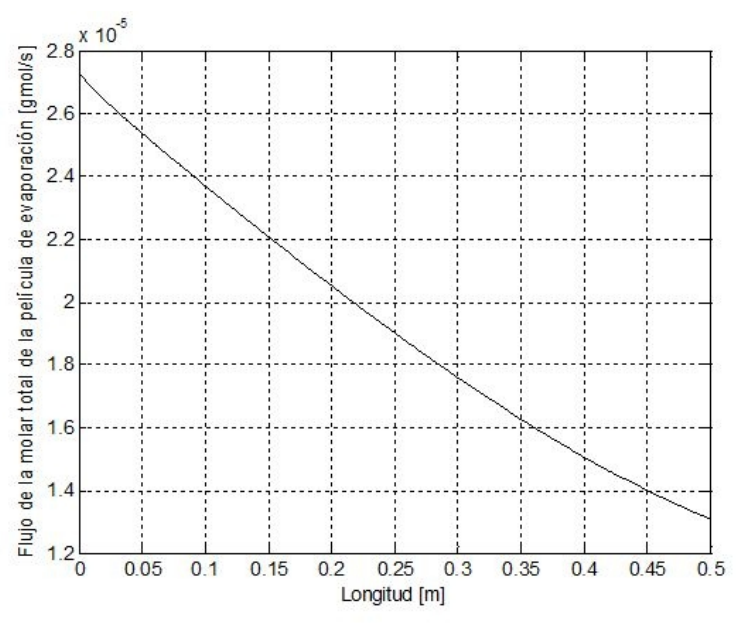

Figura 9. Flujo molar total en la película de Evaporación

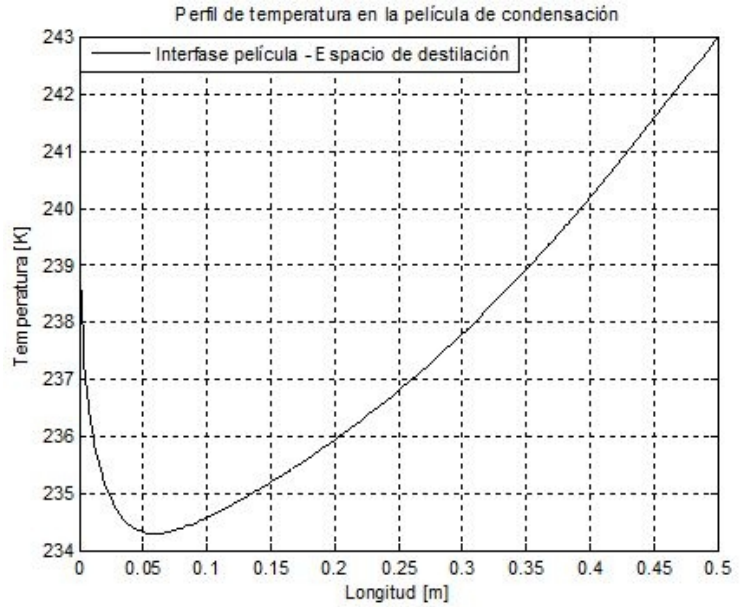

Figura 10. Perfil de temperaturas en la película de condensación

En la Figura 11 y Figura 12, se presenta el flujo molar total y el espesor de la película de condensación, los cuales aumentan a lo largo del destilador molecular, debido a la ganancia de la masa que se evapora y se condensa en la pared de condensación.

En el perfil de concentraciones de la película de condensación (Figura 13), se puede observar que el proceso se lleva a cabo principalmente para separar el limoneno y concentrar el aceite esencial de cidrón, ya que este terpeno le brinda inestabilidad térmica y química al aceite, sin embargo también hay arrastre en baja concentración de compuestos de importancia para el aceite como el citral. 


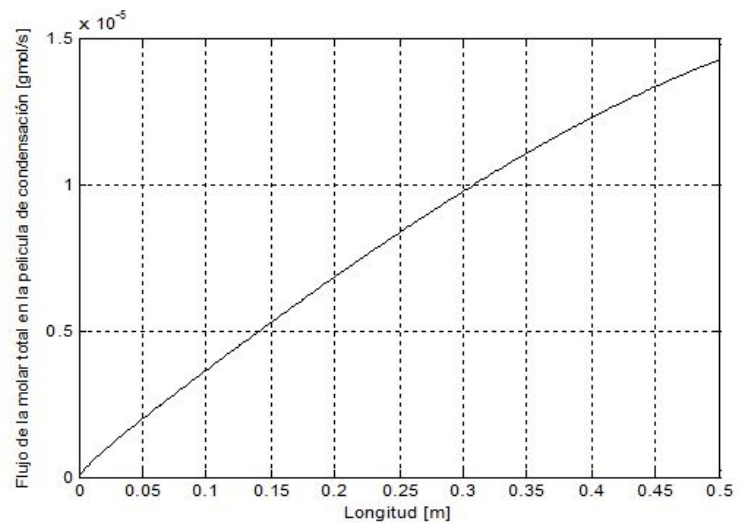

Figura 11. Flujo molar total de la película de condensación

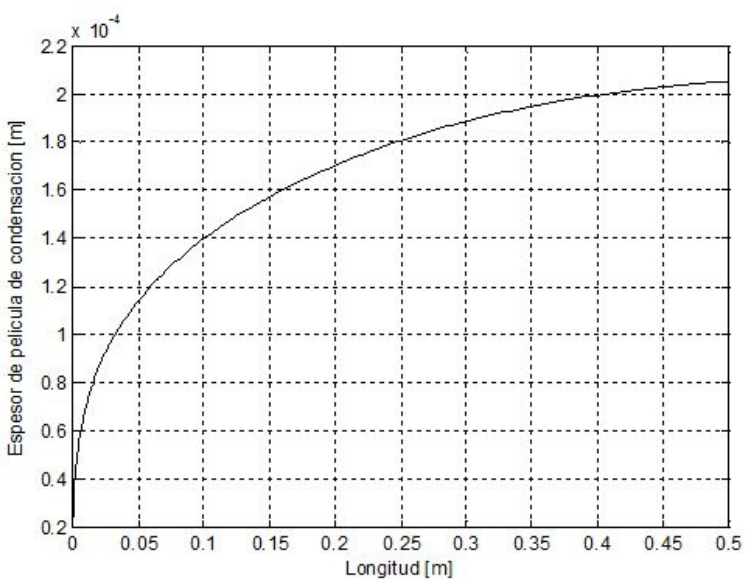

Figura 12. Espesor de la película de condensación

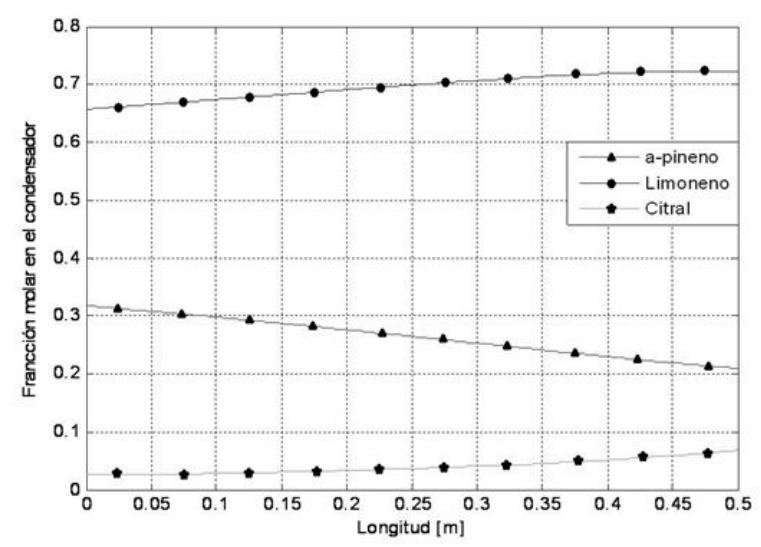

Figura 13. Perfil de concentraciones de la película de condensación
En la literatura no se reportan, estudios de concentración de aceite esencial de cidrón por destilación molecular o por otro método, lo cual impidió la comparación de los resultados obtenidos con los reportados por otros autores. Sin embargo, el comportamiento de los perfiles obtenido es similar a los reportados por otros autores para mezclas binarias Cermak et al. (2007), Martinello et al. (2007)

\section{Conclusiones}

A la vista de los resultados anteriores existe una gran similitud con el comportamiento de los perfiles de masa y energía obtenidos por otros autores a nivel matemático y experimental para la separación y purificación de mezclas binarias, por lo que queda disponible un modelo de mezclas multi-componente para realizar la concentración de aceites esenciales como herramienta fiable para la simulación de distintas condiciones de operación.

En el proceso de destilación molecular se presenta un descenso de la temperatura al inicio de la película de evaporación, debido a que la mezcla necesita energía interna para poder iniciar la evaporación. Para evitar este problema es recomendable alimentar a una temperatura cercana a la temperatura de evaporación y así mejorar la eficiencia del destilador.

Como es de esperar la composición de la sustancia menos volátil en el evaporador, aumenta a lo largo de la longitud de destilador. Se puede concluir que las temperaturas altas de evaporación aumentan la eficiencia del proceso, sin embargo se debe tener cuidado de que ésta no afecte la naturaleza de la sustancia.

La simulación de la operación de un destilador molecular para una mezcla multi-componente, permite realizar la evaluación del proceso en la etapa de diseño, convirtiéndose en una herramienta elemental a la hora de desarrollar un proceso, ya que reduce tiempo, simplifica costos y permite comparar de manera eficiente diferentes procesos, acercando los conceptos y decisiones a la realidad. 


\section{Nomenclatura}

$\Lambda=$ Trayectoria libre media $[\mathrm{m}]$

molec $r=$ Radio molecular $[\mathrm{m}]$

$\mathrm{Nm}=$ Numero de moléculas por unidad de volumen [moléculas $/ \mathrm{m} 3$ ]

$D_{i}=$ Coeficiente de difusión del componente i en la película $[\mathrm{m} 2 / \mathrm{s}]$

$\mathrm{F}=$ Flujo molar total en el punto de alimentación de la película sobre la superficie de evaporación [gmol/s]

$\mathrm{g}=$ Aceleración debida a la gravedad $[9.8 \mathrm{~m} / \mathrm{s} 2]$

$\Delta H_{\text {evp } i}=$ Calor latente de vaporización del componente i en la película [J/gmol]

$M_{i}=$ Masa molecular del componente $\mathrm{i}[\mathrm{g} / \mathrm{gmol}]$

$\mathrm{r}=$ radio del cilindro de evaporación $[\mathrm{m}]$

$\mathrm{R}=$ Constante de los gases ideales $[8.314 \mathrm{~m} 3 \mathrm{~Pa} /$ $(\mathrm{gmol} \mathrm{K})]$

$\mathrm{z}=$ coordenada axial $[\mathrm{m}]$

$\Gamma=$ Conductividad térmica de la mezcla $[\mathrm{W} /(\mathrm{m}$ $\mathrm{K})]$

$h_{1}=$ Espesor de la película $[\mathrm{m}]$

$\vartheta=$ Viscosidad cinemática de la película [m2/s]

$T=$ Temperatura $[\mathrm{K}]$

$v_{z}=\operatorname{velocidad}[\mathrm{m} / \mathrm{s}]$

$\rho=\operatorname{densidad}[\mathrm{kg} / \mathrm{m} 3]$

$C p=$ Capacidad calorífica $[\mathrm{J} / \mathrm{kg} \mathrm{K}]$

$T_{f}=$ Temperatura de alimentación $[\mathrm{K}]$

$T w=$ Temperatura de la pared $[\mathrm{K}]$

$p_{o}=$ Presión de vapor saturado
$T s=$ Temperatura de la superficie $[\mathrm{K}]$

$k=$ Velocidad de evaporación $\left[\mathrm{mol} / \mathrm{m}^{2} \mathrm{~s}\right]$

$C_{i}=$ Concentracción de i $\left[\mathrm{mol} / \mathrm{m}^{3}\right]$

$D_{i}=$ coeficiente de difussion de $\mathrm{i}\left[\mathrm{m}^{2} / \mathrm{s}\right]$

$C_{i o}=$ Concentración de $\mathrm{i}$ en la alimentación $\left[\mathrm{mol} / \mathrm{m}^{3}\right]$

$I_{i}=$ Velocidad de flujo de i $[\mathrm{mol} / \mathrm{s}]$

$\mu=$ viscosidad de liquido

$\mathrm{V}_{1}=$ Volumen molar de la especie $1[\mathrm{~cm} 3 / \mathrm{mol}]$

$\mu_{2}=$ Viscosidad de la especie $2[m \cdot P a s=c p]$

$\bigoplus_{12}^{o}=$ Difusividad de la especie 1 diluido infinitesimalmente en la especie $2\left[\mathrm{c} \mathrm{m}_{2} / \mathrm{s}\right]$

\section{Referencias bibliográficas}

Argyropoulou, C., Daferera, D., Tarantilis, P.A., Fasseas, C. \& Polissiou, M. (2007). Chemical composition of the essential oil from leaves of Lippia citriodora H.B.K. (Verbenaceae) at two developmental stages. Biochemical Systematics and Ecology (12), 831-837.

Batistella, C.B. \& Maciel, M.R.W. (1996). Modeling, simulation and analysis of molecular distillators: Centrifugal and falling film. Computers \& Chemical Engineering (Supplement 1), S19-S24.

Bhandarkar, M. \& Ferron, J.R. (1991). Simulation of rarefied vapor flows. Industrial \& Engineering Chemistry Research (5), 998-1007.

Bilia, A.R., Giomi, M., Innocenti, M., Gallori, S. \& Vincieri, F.F. (2008). HPLC-DAD-ESI-MS analysis of the constituents of aqueous preparations of verbena and lemon verbena and evaluation of the antioxidant activity. Journal of Pharmaceutical and Biomedical Analysis (3), 463-470. 
Bose, A. \& Palmer, H.J. (1984). Influence of heat and mass transfer resistances on the separation efficiency in molecular distillations. Industrial \& Engineering Chemistry Fundamentals (4), 459465.

Cardona, C.A., Gómez, Á., Ramírez, J. \& Rojas, F. (2005). Modelado y simulación de una destilación molecular de película descendente. Ingeniería Química (426), 98-112.

Carnat, A., Carnat, A.P., Fraisse, D. \& Lamaison, J.L. (1999). The aromatic and polyphenolic composition of lemon verbena tea. Fitoterapia (1), 44-49.

Cermak, S.C., John, A.L. \& Evangelista, R.L. (2007). Enrichment of decanoic acid in cuphea fatty acids by molecular distillation. Industrial Crops and Products (1), 93-99.

Cerón, I.X. (2009). Separación de metabolitos de los aceites esenciales de eucalipto y cidrón por destilación molecular. Magister en Ingeniería Ingeniería Química, Ingeniería Química, Universidad Nacional de Colombia, Manizales.

Cvengros, J., Lutisan, J. \& Micov, M. (2000). Feed temperature influence on the efficiency of a molecular evaporator. Chemical Engineering Journal(1), 61-67.

Chang, S.S., Ostric-Matijasevic, B., Hsieh, O.A.L. \& Huang, C.-L. (1977). Natural antioxidants from Rosemary and Sage. Journal of Food Science (4), 1102-1106.

Lutisan, J. \& Cvengros, J. (1995). Mean free path of molecules on molecular distillation. The Chemical Engineering Journal and the Biochemical Engineering Journal (2), 39-50.

Lutisan, J., Cvengros, J. \& Micov, M. (2002). Heat and mass transfer in the evaporating film of a molecular evaporator. Chemical Engineering Journal (2-3), 225-234.

Marrero, J. \& Gani, R. (2001). Groupcontribution based estimation of pure component properties. Fluid Phase Equilibria (0), 183-208.
Martinello, M., Hecker, G. \& Carmen Pramparo, M.d. (2007). Grape seed oil deacidification by molecular distillation: Analysis of operative variables influence using the response surface methodology. Journal of Food Engineering (1), 60-64.

Martinello, M.A., Bonino, F., Gatica, E.A. \& Pramparo, M.D.C. (2003). Modelado y análisis de la destilación molecular de película descendente. Mecánica Computacional (19), 1692-1701.

Martins, P.F., Ito, V.M., Batistella, C.B. \& Maciel, M.R.W. (2006). Free fatty acid separation from vegetable oil deodorizer distillate using molecular distillation process. Separation and Purification Technology (1), 78-84.

Micov, M., Lutišan, J. \& Cvengroš, J. (1997). Balance Equations for Molecular Distillation. Separation Science and Technology (18), 3051 3066.

Nguyen, A.-D. \& Le-Goffic, F. (1997). Limits of wiped film short-path distiller. Chemical Engineering Science (16), 2661-2666.

Ragone, M.I., Sella, M., Conforti, P., Volonté, M.G. \& Consolini, A.E. (2007). The spasmolytic effect of Aloysia citriodora, Palau (South American cedrón) is partially due to its vitexin but not isovitexin on rat duodenums. Journal of Ethnopharmacology (2), 258-266.

Sales-Cruz, M. \& Gani, R. (2006). ComputerAided Modelling of Short-Path Evaporation for Chemical Product Purification, Analysis and Design. Chemical Engineering Research and Design (7), 583-594.

Shao, P., Jiang, S.T. \& Ying, Y.J. (2007). Optimization of Molecular Distillation for Recovery of Tocopherol from Rapeseed Oil Deodorizer Distillate Using Response Surface and Artificial Neural Network Models. Food and Bioproducts Processing (2), 85-92.

Vekiari, S.A., Protopapadakis, E.E., Papadopoulou, P., Papanicolaou, D., Panou, C. \& 
Vamvakias, M. (2001). Composition and Seasonal Variation of the Essential Oil from Leaves and Peel of a Cretan Lemon Variety. Journal of Agricultural and Food Chemistry (1), 147-153. 